\title{
Intradural chordoma mimicking a lateral sphenoid wing meningioma: a case report
}

\author{
Przemysław Kunert', Tomasz Dziedzic ${ }^{1}$, Ewa Matyja², Andrzej Marchel ${ }^{1}$ \\ 1Department of Neurosurgery, Medical University of Warsaw, ${ }^{2}$ Department of Experimental and Clinical Neuropathology, \\ M. Mossakowski Medical Research Centre, Polish Academy of Sciences, Warsaw, Poland
}

\begin{abstract}
Chordomas are rare tumours arising from notochordal remnants. Classical chordomas are generally extradural and, despite benign histopathology, they typically destroy the clivus and surrounding bone structures. Intradural lesions are extremely rare and less than thirty cases of intracranial, exclusively intradural chordomas have been reported so far. The intracranial, intradural but extranotochordal location of chordoma is extremely unique. The authors present a case of chordoma in intracranial location that clinically mimics lateral sphenoid wing meningioma. A previously healthy 39-year-old man was admitted to our Department because of optic disc oedema without neurological deficits. Neuroimaging studies showed a large, contrast-enhanced tumour in the right frontotemporal region that was thought to be a pterional meningioma. The patient underwent successful removal of the tumour. Histopathological study revealed a typical pattern of chordoma, confirmed by immunohistochemical findings. Because of the tumour location the differentiation between chordoma and chordoid meningioma ought to be considered. Such cases, including the present one, may lead to the conclusion that embryonic notochordal remnants may be lost in different places, even away from the neuroaxis.
\end{abstract}

Key words: chordoma, intradural, extraosseous, differential diagnosis.

\section{Introduction}

Chordomas are rare, aggressive tumours that arise from notochordal remnants. They constitute about $1 \%$ of all intracranial tumours and the reported incidence rate is 1 per 1000000 per year. Chordomas are located in the skull base in approximately $1 / 3$ of cases [14]. Classical chordomas are generally extradural and, although they are histopathologically benign, they typically destroy the clivus and surrounding bone structures. The intracranial, intradural but extranotochordal location of chordoma is extremely rare and there are currently less than thirty reported cases of intracranial, exclusively intradural chordomas without bone invasion $[3,7,24]$. The majority of these lesions were located in the prepontine space. Intradural extraosseous chordomas have occasionally been found within the brain, in hypothalamic and pineal regions $[8,10]$. Intradural chordomas are believed to be less aggressive and have a better prognosis for patients. They are presumably a transitional form between a benign notochordal hamartomatous remnant (ecchordosis physaliphora) and a typical chordoma [6,19]. We present a unique case of an intradural, extraosseous chordoma in an intracra- 
nial location that clinically mimics lateral sphenoid wing meningioma.

\section{Case report}

\section{Clinical presentation}

A previously healthy 39-year-old man was admitted to our Department of Neurosurgery because of a bilateral optic disc oedema without decreased visual acuity. It was discovered during a routine ophthalmological examination performed during a periodic exam commissioned by an occupational medicine physician. On admission, the patient was in good general health. The neurological examination revealed no abnormalities. A non-contrast computed tomography scan revealed a hypodense lesion in the right frontotemporal region, causing mass effect. The tumour size was $70 \times 40 \times 75 \mathrm{~mm}$. The computed tomography with bone setting showed no structural changes except for a suspicion of insignificant hyperostosis at the tumour attachment site. Right external carotid angiography demonstrated a mass attached to the pterion and supplied by the middle meningeal artery. MRI scans identified that the tumour was hypointense on T1weighted images and hyperintense on T2-weighted images and, following contrast medium administration, a relatively uniform enhancement was found (Fig. 1). The tumour was thought to be a pterional meningioma and the patient underwent surgery. No macroscopic bone involvement was noted. After incision of the dura, an extra-arachnoid, highly vascularised soft tumour was found. The tumour boundaries were well defined and there was no difficulty in separating it from the arachnoid. The tumour was completely removed and the dural attachment was also excised. Duraplasty was performed using a synthetic dural substitute and the bone flap was replaced. The histopathological study of the tumour identified that it was a chordoma. The patient was discharged home with no neurological deficits. Currently, 10 months after surgery, the patient is able to carry on normal activity and work at his previous employment. The patient did not undergo radiotherapy. The follow-up MRI performed 6 months after surgery revealed no recurrence or residual tumour.

\section{Histopathological study}

The biopsied tumour tissue was fixed in $10 \%$ formalin, embedded in paraffin, and stained with hema- toxylin-eosin (H\&E). Immunohistochemical analyses were performed on paraffin-embedded specimens using the avidin-biotin complex method $(A B C)$ with $D A B$ as a chromogen, using antibodies against epithelial membrane antigen (EMA), cytokeratin cocktail AE1/AE3, S-100 protein, glial fibrillary acid protein (GFAP), vimentin, and Ki67 antigen, clone MIB-1 (all antibodies from Dako, Glostrup, Denmark).

Microscopically, the tumour displayed the typical histological pattern for classical chordoma with epithelial cells arranged in cords, clusters, or strands and embedded in an abundant, eosinophilic, mucoid matrix (Fig. 2A, B). Numerous multivacuolated cells with clear, foamy or bubbly cytoplasm, representing so-called physaliphorous cells, were present. The tumour showed apparent lobules separated by fibrous septa. The typical meningothelial aeries were not identified within the whole material studied. Immunohistochemically, the neoplastic cells showed an epithelial phenotype with reactivity for cytokeratin AE1/AE3 (Fig. 2C) and expression of EMA (Fig. 2D). In some areas the neoplastic cells were positive for S-100 protein. The tumour cells were negative for GFAP. The MIB-1 labelling index was relatively low but its focal increase was noted. The histopathological diagnosis of chordoma was based on its typical morphology, the lack of classical meningothelial features and immunohistochemical findings.

\section{Discussion \\ Radiological features}

A chordoma usually has subtle hyperdense or isodense signal in non-contrast-enhanced CT. The present case was hypodense without any calcifications or bone destruction, which is not the typical radiological appearance of a chordoma [26]. The tumour was mildly hypointense and relatively homogenously hyperintense before and after the contrast injection in T1-weighted MRI images, respectively. This strongly suggested meningioma. The lesion was highly hyperintense only in T2-weighted images, which raised doubts. Strong hyperintensity on T2-weighted images is typical of chordomas [18]; while, meningiomas are usually isointense or mildly hyperintense.

A "dural tail sign" is a typical feature of meningiomas on contrast-enhanced MR images; although, it has been not considered as pathognomonic for many years. Many other intracranial lesions are occasionally accompanied by a dural tail, including: schwanno- 


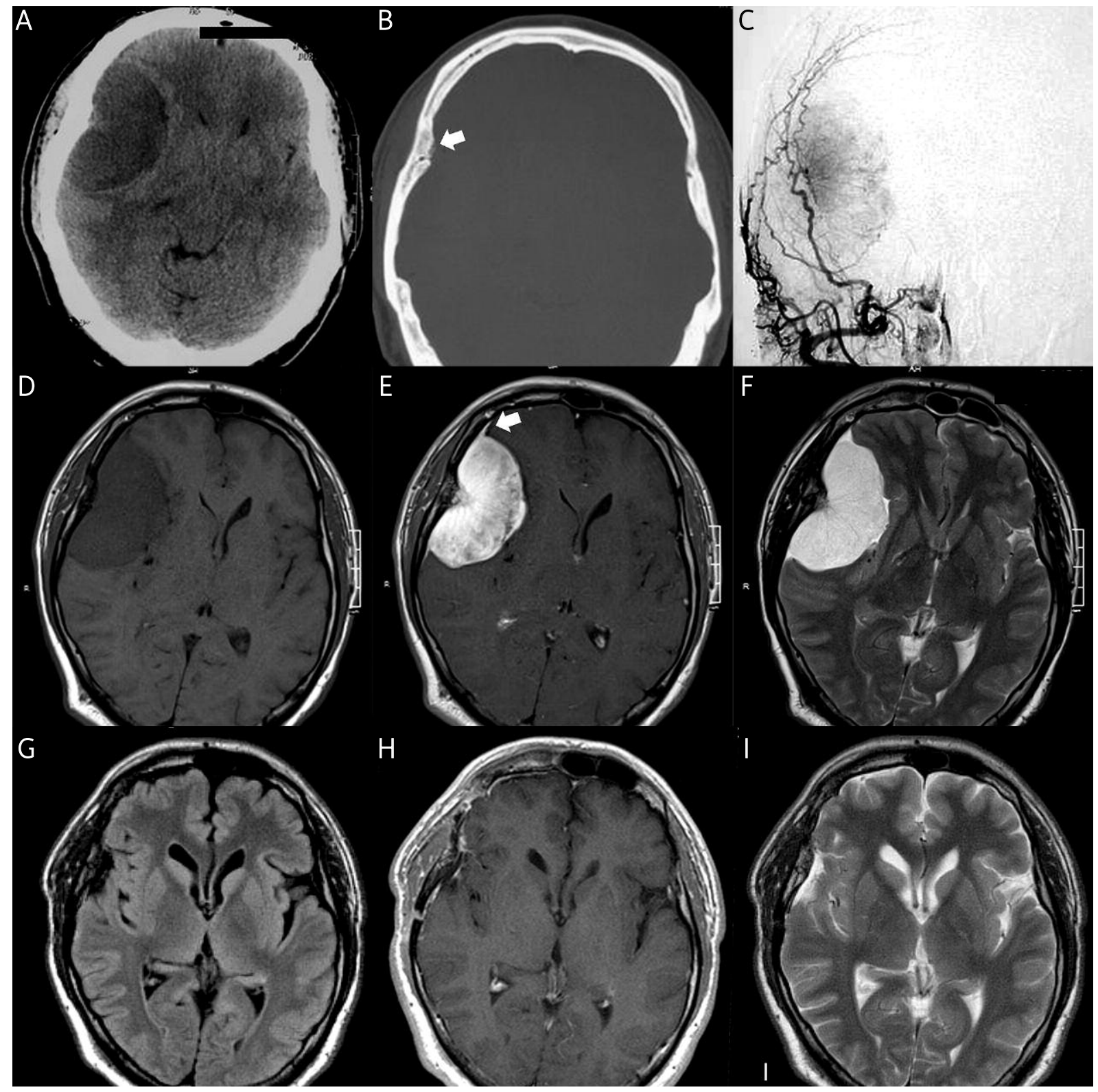

Fig. 1. A, B) CT examination of the skull and brain without contrast revealed a hypodense tumour attached to the pterion with a suspicion of some endostosis (white arrow). The spongy bone is not invaded by the tumour. C) External carotid artery angiography showed the typical vascular supply for a lateral sphenoid wing meningioma. D-F) Preoperative MRI of the brain. D, E) The T1-weighted MR image showed a meningioma-like mass, with a 'dural tail sign' (white arrow). F) Only the hyperintense signal of the lesion in the T2-weighted sequence suggested another entity. G-I) Follow-up MRI images obtained 6 months after surgery revealed no regrowth or residual tumour.

mas, gliomas, metastases, lymphomas, myelomas, chloromas, pituitary tumours, and granulomatosis [11]. To our knowledge, the coexistence of dura enhancement around the chordoma has been described in only one case [16].

\section{Clinical considerations}

The age of our patient was typical of a chordoma, not of a meningioma. The mean age of patients with skull base chordomas in the series of al-Mefty and Borba was 38 years [2]. Chordomas, even if intradural, 
arise along the neuroaxis. Reports of other locations are extremely rare. Ectopic chordomas were found in the maxillary sinus and soft tissues of the neck $[5,22,23]$. To our knowledge, an intradural, but extranotochordal, location of a chordoma has only been reported in exceptional cases. The first case of an intracerebral hypothalamic chordoma was reported by Commins et al. in 1994 [8]. The second case has been recently described by Figueiredo et al. [10]. It was a pineal chordoma with abdominal metastasis, due to previous ventriculoperitoneal shunt placement. The origin of such intraparenchymal tumours is not clear and it has been suggested that they arise from ecchordosis physaliphora (EP) or directly from notochord remnants that subsist within brain tissue $[8,10]$. The cases described suggest ectopic notochordal remnants may be located in different areas, even away from the neuroaxis.

\section{Histopathological considerations}

The histopathological diagnosis of a chordoma is based on its typical morphology, including epithelial features, the presence of distinct physaliphorous cells, and immunopositivity for epithelial markers (CKs and EMA) and S-100 protein. Other lesions, including ecchordosis physaliphora and tumours of chordoma-like morphology, such as chordoid meningioma, chordoid gliomas, or metastatic mucinous carcinoma, ought to be considered in the differential diagnosis. The distinction of a chordoma from EP is particularly difficult as both lesions exhibit similar histopathological, immunohistochemical, and ultrastructural characteristics [27]. Nevertheless, EP represents a slow-growing, non-neoplastic, usually asymptomatic, notochord lesion of a small size, ranging from a few millimetres to $2 \mathrm{~cm}$ [4]. EP usually displays a low
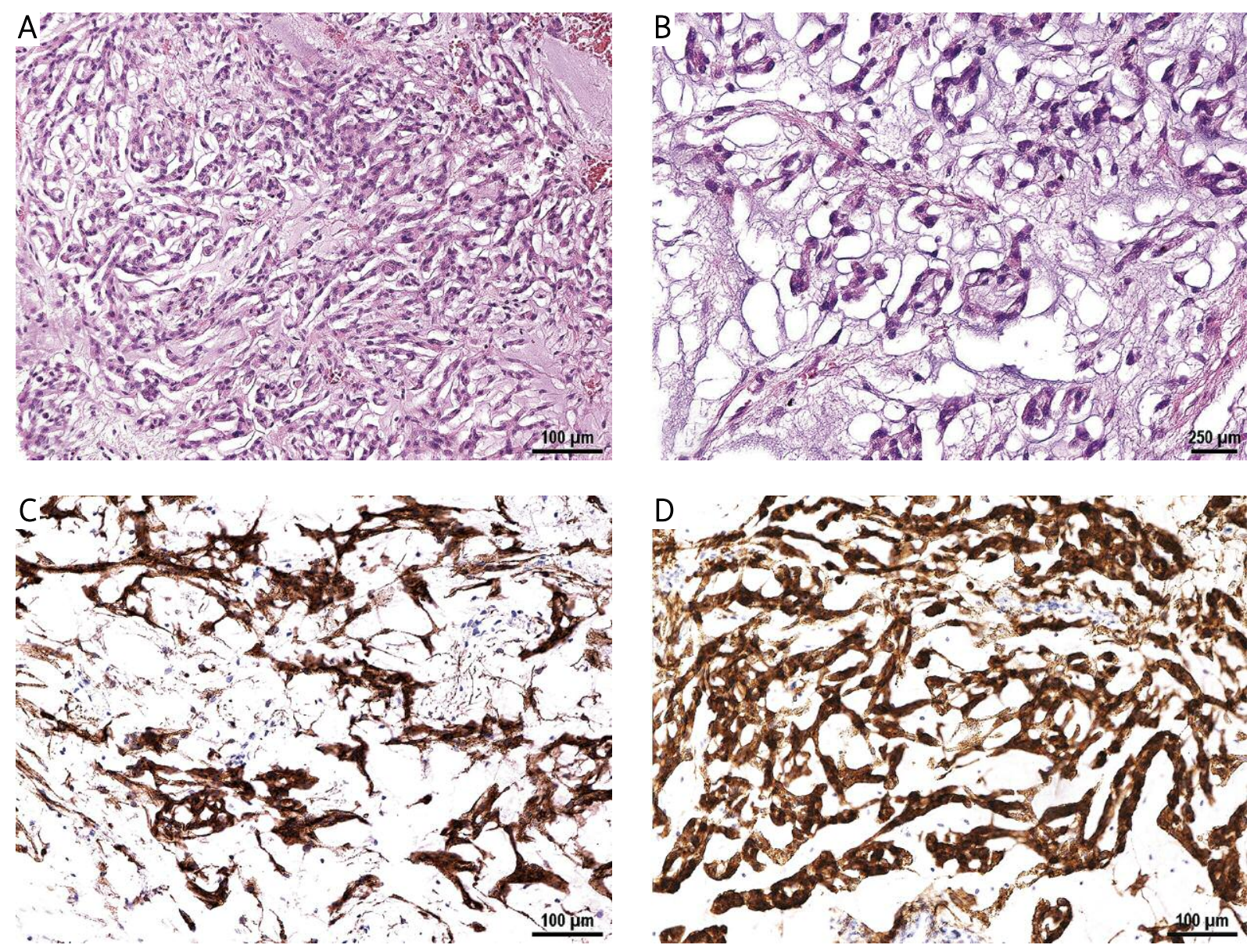

Fig. 2. Morphology of the chordoma. A) Cords of epithelioid cells in a myxoid background. B) Tumour cells with vacuolated, clear, or bubbly cytoplasm, representing so-called physaliphorous cells. C) Immunostaining for AE1/AE3. D) Immunoreactivity for EMA. Bars: A, C, D - $100 \mu \mathrm{m} ; \mathrm{B}-250 \mu \mathrm{m}$. 
Ki67 proliferation index [7]; thus, when intradural chordoma is suspected, staining for MIB-1 might be helpful in the differential diagnosis [20]. Moreover, on MRI scans, EP presents as a non contrast-enhanced retroclival mass [7,15,27]; thus, this lesion could be excluded in our case.

The main histopathological features of a chordoma and chordoid meningioma are very similar apart from so-called physaliphorous cells, which are typical of a classical chordoma. The correct diagnosis might be confirmed by immunohistochemical studies. A chordoma is immunopositive for cytokeratin, S-100, vimentin and EMA; whereas, a chordoid meningioma is positive for EMA and vimentin but mostly negative for cytokeratin and S-100 protein $[13,21]$. Moreover, in the majority of chordoid meningiomas, there are distinctive areas of a conventional type of meningioma. In addition, chordomas and chordoid gliomas resemble each other histologically; however, a chordoid glioma is a GFAP immunopositive tumour [21]. A chordoma should also be distinguished from a metastatic mucinous adenocarcinoma, which is usually immunonegative for vimentin and S-100 protein. It has been postulated that an intradural chordoma should be distinguished as a separate entity because of its differences from an intraosseous chordoma and similarities to EP $[1,6,7,19,25]$. In doubtful cases the diagnosis of chordoma might be supported by expression of notochordal marker Brachyury, which is proved to be a specific marker for chordoma.

\section{Therapeutic considerations}

Currently, we recommend using proton beam therapy after chordoma removal, but a "wait-and-see" approach was preferred for this patient. Based on neurosurgical literature, intradural chordomas seem to have less aggressive behaviour than intraosseous ones. Long-term survival after complete tumour resection has been reported $[9,25]$. This can be partly explained by the expansion pattern, which has a greater chance for total resection. In our case, we encountered no difficulties in the dissection of the tumour from the arachnoid membrane and the dural attachment could be easily separated from the bone. However, even if the resection was incomplete and radiation therapy was not applied, long-term survival without disease progression is possible $[3,17]$. Therefore, the radiation therapy should not be applied directly after surgery to avoid overtreatment [19]. However, two cases of intradural chordoma with a more aggressive nature have recently been described [12]. Although intradural chordomas are generally benign, long-term follow-up of these patients has only been described for two cases; thus, prolonged close observation is essential $[7,9,17]$.

The intracranial, intradural, extranotochordal location of a chordoma is extremely rare. Such cases suggest that embryonic notochordal remnants may be lost in different areas, even away from the neuroaxis. The biological behaviour of ectopic chordomas remains unknown; thus, it is essential that patients are closely followed after surgical removal of these chordomas.

\section{References}

1. Adamek D, Malec M, Grabska N, Krygowska-Wajs A, Gałązka K. Ecchordosis physaliphora - a case report and a review of notochord-derived lesions. Neurol Neurochir Pol 2011; 45: 169-173.

2. al-Mefty O, Borba LA. Skull base chordomas: a management challenge. J Neurosurg 1997; 86: 182-189.

3. Bergmann M, Abdalla Y, Neubauer U, Schildhaus HU, ProbstCousin S. Primary intradural chordoma: report on three cases and review of the literature. Clin Neuropathol 2010; 29: 169-176.

4. Bhat DI, Yasha M, Rojin A, Sampath S, Shankar SK. Intradural clival chordoma: a rare pathological entity. J Neurooncol 2010; 96 : 287-290.

5. Bumber Z, Krizanac S, Janjanin S, Bilic M, Bumber B. Extranotochordal extralaryngeal chordoma: a case report. Auris Nasus Larynx 2009; 36: 116-119.

6. Chang SW, Gore PA, Nakaji P, Rekate HL. Juvenile intradural chordoma: case report. Neurosurgery 2008; 62: 525-526.

7. Ciarpaglini R, Pasquini E, Mazzatenta D, Ambrosini-Spaltro A, Sciarretta V, Frank G. Intradural clival chordoma and ecchordosis physaliphora: a challenging differential diagnosis: case report. Neurosurgery 2009; 64: 387-388.

8. Commins D, Baran GA, Molleston M, Vollmer D. Hypothalamic chordoma. Case report. J Neurosurg 1994; 81: 130-132.

9. Danilewicz B, Danilewicz M, Czepko R, Stachura K. Intradural clivus chordoma: surgical treatment with no tumor regrowth 12 years after surgery. Przegl Lek 2000; 57: 178-180.

10. Figueiredo EG, Tavares WM, Welling L, Rosemberg S, Teixeira MJ. Ectopic pineal chordoma. Surg Neurol Int 2011; 2: 145.

11. Guermazi A, Lafitte F, Miaux Y, Adem C, Bonneville JF, Chiras J. The dural tail sign - beyond meningioma. Clin Radiol 2005; 60: 171-188.

12. Ito E, Saito K, Nagatani T, Ishiyama J, Terada K, Yoshida M, Wakabayashi T. Intradural cranial chordoma. World Neurosurg 2010; 73: 194-197.

13. Matyja E, Grajkowska W, Lazarczyk M, Marchel A, Czernicki T. Chordoid meningiomas of a different histopathological pattern: a report of two cases. Folia Neuropathol 2006; 44: 34-41.

14. McMaster ML, Goldstein AM, Bromley CM, Ishibe N, Parry DM. Chordoma: incidence and survival patterns in the United States, 1973-1995. Cancer Causes Control 2001; 12: 1-11. 
15. Mehnert F, Beschorner R, Kuker W, Hahn U, Nagele T. Retroclival ecchordosis physaliphora: MR imaging and review of the literature. AJNR Am J Neuroradiol 2004; 25: 1851-1855.

16. Nakasu Y, Nakasu S, Ito R, Mitsuya K, Fujimoto O, Saito A. Tentorial enhancement on MR images is a sign of cavernous sinus involvement in patients with sellar tumors. AJNR Am J Neuroradiol 2001; 22: 1528-1533.

17. Nishigaya K, Kaneko M, Ohashi Y, Nukui H. Intradural retroclival chordoma without bone involvement: no tumor regrowth 5 years after operation. Case report. J Neurosurg 1998; 88: 764-768.

18. Nishiguchi T, Mochizuki K, Ohsawa M, Inoue T, Kageyama K, Suzuki A, Takami T, Miki Y. Differentiating benign notochordal cell tumors from chordomas: radiographic features on MRI, CT, and tomography. AJR Am J Roentgenol 2011; 196: 644-650.

19. Riopel C, Michot C. Chordomas. Ann Pathol 2007; 27: 6-15.

20. Roberti F, Sekhar LN, Jones RV, Wright DC. Intradural cranial chordoma: a rare presentation of an uncommon tumor. Surgical experience and review of the literature. J Neurosurg 2007; 106: 270-274.

21. Sangoi AR, Dulai MS, Beck AH, Brat DJ, Vogel H. Distinguishing chordoid meningiomas from their histologic mimics: an immunohistochemical evaluation. Am J Surg Pathol 2009; 33: 669-681.

22. Shugar JM, Som PM, Krespi YP, Arnold LM, Som ML. Primary chordoma of the maxillary sinus. Laryngoscope 1980; 90: 18251830.

23. Sivakumar S. Primary extranotochordal cervical chordoma masquerading as a thyroid neoplasm: a case report. Acta Cytol 2010; 54: 823-828.

24. Steenberghs J, Kiekens C, Menten J, Monstrey J. Intradural chordoma without bone involvement. Case report and review of the literature. J Neurosurg 2002; 97: 94-97.

25. Wolfe JT 3rd, Scheithauer BW. "Intradural chordoma" or "giant ecchordosis physaliphora"? Report of two cases. Clin Neuropathol 1987; 6: 98-103.

26. Woźnica J, Kendall B, Bryc S, Losicki M, Kingsley D. Value of CT and NMR imaging in diagnosing of chordomas. Ann Univ Mariae Curie Sklodowska Med 1990; 45: 181-186.

27. Wyatt RB, Schochet SS Jr, McCormick WF. Ecchordosis physaliphora. An electron microscopic study. J Neurosurg 1971; 34: 672-677. 